\title{
MLN4924 neddylation inhibitor promotes cell death in paclitaxel-resistant human lung adenocarcinoma cells
}

\author{
QIANG XU ${ }^{1 *}$, GUIBIN LIN ${ }^{2 *}$, HUIZHE XU ${ }^{2}$, LULU HU $^{2}$, YUPENG WANG $^{2}$, SHA DU $^{2}$, \\ WUGUO DENG ${ }^{2}$, WENXIAN HU ${ }^{3}$, WEI CHENG ${ }^{2}$ and $\mathrm{KE} \mathrm{JIANG}^{2}$
}

\begin{abstract}
${ }^{1}$ Department of Orthopedics, The Affiliated Yuhuangding Hospital of Qingdao University Medical College, Yantai, Shandong 264000; ${ }^{2}$ Institute of Cancer Stem Cell, Dalian Medical University Cancer Center, Dalian, Liaoning 116044; ${ }^{3}$ Key Laboratory of Biotherapy of Zhejiang Province, Department of Surgical Oncology, Sir Run Run Shaw Hospital, College of Medicine, Zhejiang University, Hangzhou, Zhejiang 310020, P.R. China
\end{abstract}

Received June 22, 2016; Accepted March 30, 2017

DOI: 10.3892/ol.2017.7314

\begin{abstract}
Acquired resistance to first-line chemotherapeutics, including paclitaxel (PTX), is a primary factor contributing to chemotherapy failure in non-small cell lung cancer (NSCLC) patients. Previous studies have identified that targeting NEDD8-activating enzyme (NAE) with MLN4924 effectively overcomes platinum resistance in preclinical models of ovarian cancer. However, the underlying mechanisms are yet to be fully elucidated. The present study demonstrates that the inhibition of the neddylation pathway with MLN4924 an NAE inhibitor inhibited protein neddylation, inactivated cullin-RING E3 ligase and exhibited a potent antiproliferative effect on PTX-resistant A549 and H460 cells (A549/PTX and H460/PTX). The application of MLN4924 promotes apoptosis and DNA damage in A549/PTX and H460/PTX cells. Additionally, MLN4924 abrogated the 3-dimensional growth potential of these cells and inhibited the formation of the A549/PTX and H460/PTX spheroids. Notably, combining MLN4924 with PTX did not exhibit synergy in PTX-resistant NSCLC cells. Taken together, the results of the current study suggest that MLN4924 may be utilized as an effective strategy for the treatment of PTX-resistant NSCLC.
\end{abstract}

\section{Introduction}

Lung cancer is a leading cause of cancer mortality globally, in which the predominant subtype of non-small cell lung

Correspondence to: Dr Ke Jiang or Dr Wei Cheng, Institute of Cancer Stem Cell, Dalian Medical University Cancer Center, 9 Lvshun Road South, Dalian, Liaoning 116044, P.R. China

E-mail: jiangke.3@163.com

E-mail:wcheng@dmu.edu.cn

${ }^{*}$ Contributed equally

Key words: MLN4924, neddylation, paclitaxel, lung cancer, drug resistance, spheroids cancer (NSCLC) represents $\sim 80 \%$ of all cases (1). Despite advances in therapy, the overall 5-year survival rate is $<15 \%$ in patients with NSCLC (2). Currently, conventional chemotherapy remains an important treatment option for patients with NSCLC. Anti-mitotic agents, including docetaxel and paclitaxel (PTX), are predominantly used as chemotherapy regimens for NSCLC (3). However, taxane-based therapy is disadvantaged by the rapid emergence of acquired resistance (4). Therefore, novel therapeutic strategies that overcome the resistance to taxane-based therapy are required.

Nedd8 (neural precursor cell expressed, developmentally downregulated 8), a 9-kDa small ubiquitin-like molecule, is involved in protein neddylation initiated by NEDD8 activating enzyme (NAE) (5). Nedd8 serves a role in the activation of the cullin-RING ligases (CRL), also termed SKP1-cullin-F-box (SCF) E3 ligases for its founding member (CRL/SCF) (6), which has been established to be involved in the regulation of multiple DNA replication and repair pathways $(7,8)$. MLN4924 is a recently identified small molecule inhibitor of NAE and is currently in Phase I clinical trials $(9,10)$. By inhibiting neddylation, MLN4924 promotes uncontrolled S-phase DNA replication as well as in the induction of DNA damage and subsequent cell death (11-13). Therefore, MLN4924 exhibits potent antitumor activity in numerous types of cancer (14). Notably, previous studies have indicated that MLN4924 overcomes platinum resistance in preclinical models of ovarian cancer $(15,16)$, suggesting that inhibiting neddylation with MLN4924 may be a novel strategy to target drug resistance in cancer.

The focus of the present study was to investigate the effects of MLN4924 on PTX-resistant NSCLC cells. The results identified that MLN4924 suppresses the growth of PTX-resistant NSCLC cells by inducing apoptosis and DNA damage.

\section{Materials and methods}

Cell lines and cell culture. PTX-resistant H460 (H460/PTX) cells were cultured in RPMI 1640 (Gibco; Thermo Fisher Scientific Inc., Waltham, MA, USA) containing $70 \mathrm{nM}$ (60 ng/ml) PTX (Zhejiang University, Hangzhou, China) (17), PTX-resistant A549 (A549/PTX) cells, were kindly provided 
by Dr Sang Kook Lee (Seoul National University, Seoul, Korea) (18) and cultured in RPMI 1640 containing $117 \mathrm{nM}$ $(100 \mathrm{ng} / \mathrm{ml})$ PTX to maintain resistance at $37^{\circ} \mathrm{C}$ in an atmosphere containing $5 \% \mathrm{CO}_{2}$. The cells were cultured in complete media without PTX for 3 days prior to performing experiments.

Antibodies and reagents. The antibody against $\beta$-actin was purchased from Sigma-Aldrich (Merck KGaA, Darmstadt, Germany, A5316). The antibody against regulated in development and DNA damage responses 1 (REDD1) was purchased from ProteinTech Group, Inc. (Chicago, IL, USA; cat. no. 10638-1-AP). The anti-checkpoint kinase 2 (CHK2; cat. no. ab109413), anti-Histone H2AX (H2AX) (cat. no. ab124781) and anti-p21 (cat. no. ab109199) antibodies were obtained from Abcam (Cambridge, UK). The anti-cullin1 (cat. no. sc-11384) and anti-chromatin licensing and DNA replication factor 1 (CDT1; cat. no. sc-36530) antibodies were purchased from Santa Cruz Biotechnology, Inc. (Dallas, TX, USA). The following antibodies were obtained from Cell Signaling Technology, Inc. (Danvers, MA, USA): Phospho-CHK2 (cat. no. 2661), phospho-H2AX (cat. no. 9718), Wee1 (cat. no. 4936), p27 (cat. no. 3686), caspase-3 (cat. no. 9662) and poly(ADP-ribose) polymerase (PARP; cat. no. 9532). The following agents were used: MLN4924 (Merck KGaA) and PTX (Sigma-Aldrich; Merck KGaA). Recombinant human epidermal growth factor (EGF), fibroblast growth factors (FGF; both from PeproTech, Inc., Rocky Hill, NJ, USA) and B-27 (Gibco; Thermo Fisher Scientific, Inc.) were used to culture spheroids. Drugs were dissolved in dimethyl sulfoxide (DMSO) and stored at $-20^{\circ} \mathrm{C}$.

Bromodeoxyuridine (BrdU) labeling. H460/PTX and A549/PTX cells were labeled with $10 \mathrm{mmol} / 1 \mathrm{BrdU}$ (Sigma-Aldrich; Merck KGaA) in growth medium for $12 \mathrm{~h}$ at $37^{\circ} \mathrm{C}$. BrdU-labeled DNA was detected with mouse monoclonal anti-BrdU antibody (cat. no. RPN202; 1:50; GE Healthcare, Chicago, IL, USA), according to the manufacturer's protocol (19).

Colony formation assay. For clonogenic assay, cells were seeded into 6 -well plates $(300$ cell/well) in triplicate and cultured for 14 days at $37^{\circ} \mathrm{C}$ in an atmosphere containing $5 \% \mathrm{CO}_{2}$. The colonies on the plates were fixed with $4 \%$ paraformaldehyde at room temperature for $15 \mathrm{~min}$ and stained with crystal violet $(0.2 \%$ in anhydrous ethanol) at room temperature for $30 \mathrm{~min}$. Colonies with $>50$ cells were counted (19). Colonies were counted by eye and representative results of 3 independent experiments with similar results are presented.

Spheroid formation. Adherent cells were suspended in serum-free Dulbecco's modified Eagle's medium/F12 (Gibco; Thermo Fisher Scientific, Inc.) containing $20 \mathrm{ng} / \mathrm{ml}$ FGF, $20 \mathrm{ng} / \mathrm{ml}$ EGF and B-27 (B-27 and medium at a 1:50 volume ratio), plated $\left(1 \times 10^{3}\right.$ cell/well) onto a 96 -well clear flat-bottomed ultra-low attachment micro plate (Corning Incorporated, Corning, NY, USA) at $37^{\circ} \mathrm{C}$ and $5 \% \mathrm{CO}_{2}$ for 10 days. Spheroids with a diameter of $\sim 50 \mu \mathrm{m}$ were counted.

Cell death assay. A549/PTX and H460/PTX cells were suspended in complete medium for spheroids, and plated $\left(1 \times 10^{3}\right.$ cell/well) onto a 96-well clear flat-bottomed ultra-low attachment microplate (Corning Incorporated, Corning, NY, USA) at $37^{\circ} \mathrm{C}$ and $5 \% \mathrm{CO}_{2}$ for 10 days. The spheroids were treated with $10 \mu \mathrm{M}$ MLN4924 for the 24 and $72 \mathrm{~h}$ and stained with propidium iodide (PI) at $10 \mu \mathrm{g} / \mathrm{ml}$. Subsequently, cells were visualized using fluorescence microscopy as previously described (20) where red-fluorescing cells were indicative of cell death.

Western blot analysis. A549/PTX and H460/PTX cells were plated in $60-\mathrm{mm}$ dishes and treated with DMSO or 2.5, 5, 10, 20 and $0.1,0.3,1,3,10,30 \mu \mathrm{M}$ MLN4924 at $37^{\circ} \mathrm{C}$ in an atmosphere containing $5 \% \mathrm{CO}_{2}$. After 12,24 and $48 \mathrm{~h}$, cells were placed on ice, washed with cold PBS, harvested using a scraper and lysed in lysis buffer (1\% Triton X-100, $50 \mathrm{mmol} / 1$ Tris- $\mathrm{HCl}$ (pH 7.5), 150 mmol/1 NaCl, 1 mmol/l EDTA, 1 mmol/l EGTA, $5 \mathrm{mmol} / 1$ sodium pyrophosphate, $25 \mathrm{mmol} / 1 \mathrm{NaF}, 0.5 \mathrm{mmol} / 1$ sodium orthovanadate, $1 \mathrm{mmol} / 1 \mathrm{DTT}, 1 \mu \mathrm{g} / \mathrm{ml}$ pepstanin, $2 \mu \mathrm{g} / \mathrm{ml}$ leupeptin, $2 \mu \mathrm{g} / \mathrm{ml}$ aprotinin, $0.1 \mathrm{mg} / \mathrm{ml}$ phenylmethylsulfonyl fluoride). Cell lysates were centrifuged at $12,000 \mathrm{x} \mathrm{g}$ for $10 \mathrm{~min}$ at $4^{\circ} \mathrm{C}$ and supernatants were subjected to western blot analysis. Concentration of protein was determined using a bicinchoninic acid assay kit (cat. no. 23225; Pierce, Thermo Fisher Scientific, Inc.), according to the manufacturer's protocol. Protein (50 $\mu \mathrm{g}$ per lane) was loaded on SDS-PAGE (8-15\% gels) and transferred onto a nitrocellulose membrane (Applygen Technologies, Inc., Beijing, China). The membranes were blocked with $5 \%$ non-fat milk in TBST at room temperature for $3 \mathrm{~h}$ and incubated with primary antibodies at $4^{\circ} \mathrm{C}$ overnight. The antibodies for $\beta$-actin (cat. no. A5316; 1:10,000; Merck KGaA, Darmstadt, Germany,), REDD1 (1:2,000), CHK2 (1:2,000), H2AX (1:2,000), p21 (1:1,000), cullin1 (1:200), CDT1 (1:1,000), phospho-CHK2 (1:1,000), phospho-H2AX (1:1,000), Wee1 $(1: 1,000)$, p27 (1:1,000), caspase-3 $(1: 1,000)$ and PARP $(1: 1,000)$ were used. Following washing three times with TBS-Tween-20, the membranes were incubated at room temperature for $1 \mathrm{~h}$ with corresponding horseradish peroxidase-conjugated secondary antibodies (goat anti-mouse, 1:1,000, cat. no. 62-6520; goat anti-rabbit, 1:10,000, cat. no. 65-6120; Invitrogen; Thermo Fisher Scientific, Inc.). The blots were detected using an ECL Western Blot Substrate kit (cat. no. 34580; Thermo Fisher Scientific, Inc.) according to the manufacturers protocol.

Flow cytometric analysis of apoptosis. A549/PTX and H460/PTX cells were treated with DMSO or 1,5 and $10 \mu \mathrm{M}$ MLN4924 for 24,47 and $72 \mathrm{~h}$ at $37^{\circ} \mathrm{C}$ in an atmosphere containing $5 \% \mathrm{CO}_{2}$, and then the cells were harvested with $0.25 \%$ trypsin without EDTA, washed twice with ice-cold PBS and resuspended in $500 \mu \mathrm{l}$ binding buffer $(10 \mathrm{mM}$ Hepes $/ \mathrm{NaOH}$, $\mathrm{pH}$ 7.4, $140 \mathrm{mM} \mathrm{NaCl}, 2.5 \mathrm{mM} \mathrm{CaCl}$ ). Subsequently, cells were incubated with $5 \mu \mathrm{l}$ annexin $\mathrm{V}$-fluorescein isothiocyanate (40 $\mu \mathrm{g} / \mathrm{ml}$; BD Biosciences, Franklin Lakes, NJ, USA) and $5 \mu \mathrm{l}$ PI (40 $\mu \mathrm{g} / \mathrm{ml}$; BD Biosciences) in the dark for $10 \mathrm{~min}$ at room temperature and detected using flow cytometry. The percentage of apoptotic cells was determined in 3 independent experiments.

Statistical analysis. The results are expressed as means \pm standard deviation. Statistical significance was evaluated using the Student's t-test. Group comparisons were 
A

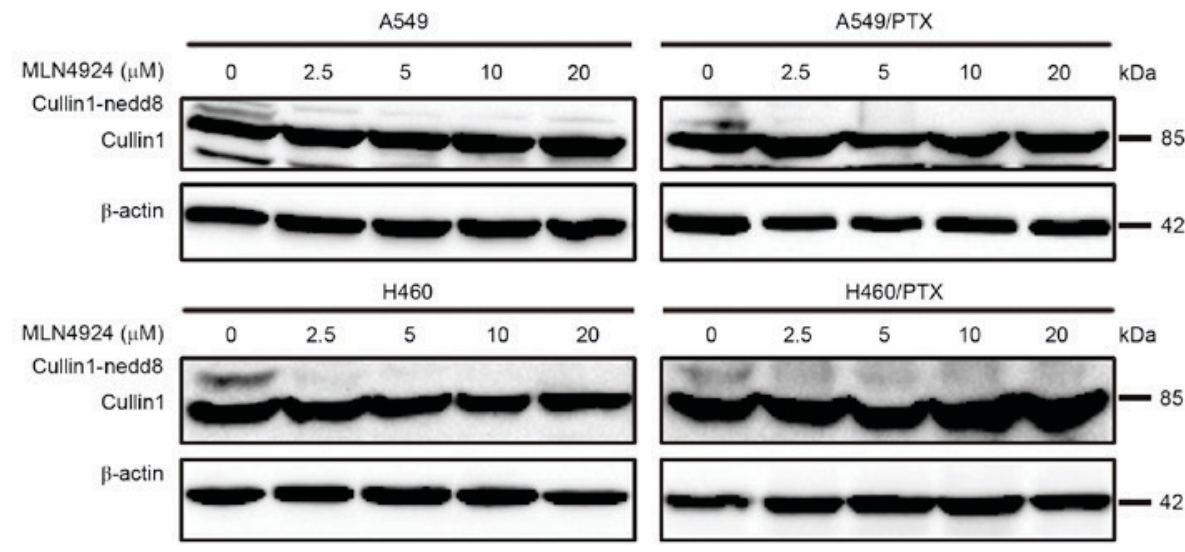

B
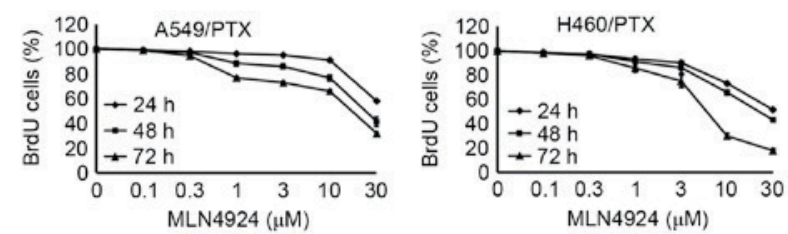

\begin{tabular}{|c|c|c|c|}
\hline $\begin{array}{r}\text { Time } \\
\text { Cell IC50 }\end{array}$ & $24 \mathrm{~h}$ & $48 \mathrm{~h}$ & $72 \mathrm{~h}$ \\
\hline A549/PTX & $18.3 \mu \mathrm{M}$ & $12.0 \mu \mathrm{M}$ & $7.3 \mu \mathrm{M}$ \\
\hline H460/PTX & $12.9 \mu \mathrm{M}$ & $10.6 \mu \mathrm{M}$ & $5.0 \mu \mathrm{M}$ \\
\hline
\end{tabular}

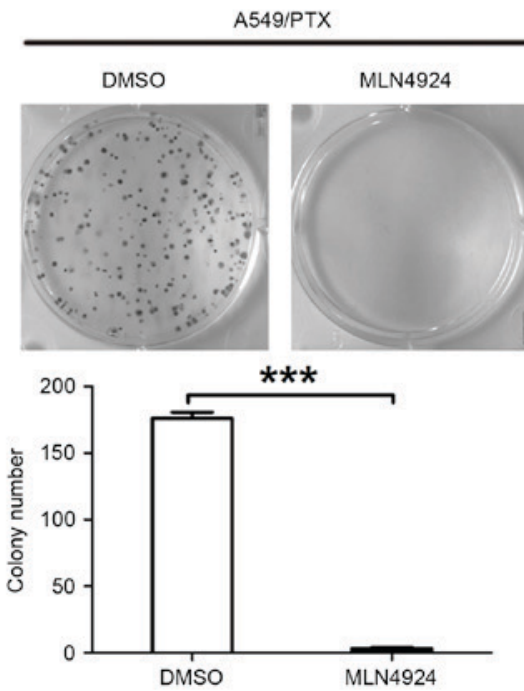

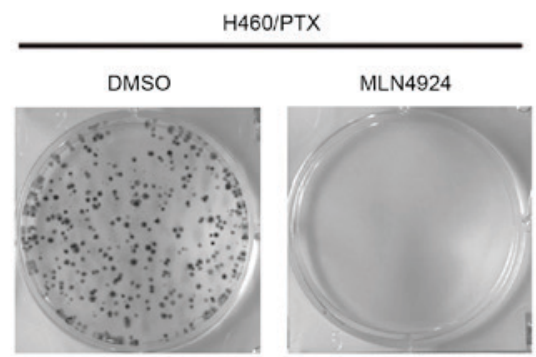

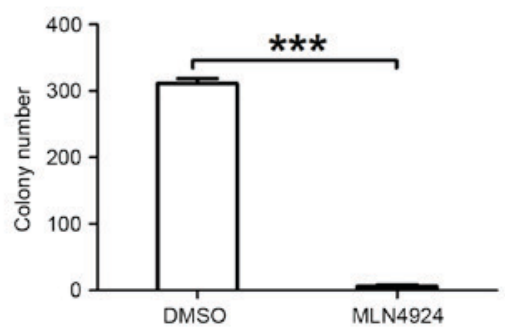

Figure 1. MLN4924 suppresses the growth of PTX-resistant NSCLC cells. (A) The A549 and H460 NSCLC cells and PTX-resistant A549 (A549/PTX) and H460 (H460/PTX) were treated with vehicle or numerous concentrations $(0,2.5,5,10$ and $20 \mu \mathrm{M})$ of MLN4924 for 24 h. (A) Cell lysates were examined for cullin1 using western blot analysis. $\beta$-actin was used as a loading control. (B) The A549/PTX and H460/PTX cells were treated with vehicle or the indicated concentrations $(0$, $0.1,0.3,1,3,10$ and $30 \mu \mathrm{M}$ ) of MLN4924 for 24,48 and $72 \mathrm{~h}$, the cell viability was determined using a BrdU assay. The results are presented as the mean \pm standard deviation. (C) The A549/PTX and H460/PTX cells were treated with vehicle or $10 \mu \mathrm{M}$ MLN4924 for 14 days in complete medium and colony formation assay was performed. The number of colonies was counted and presented as the mean \pm standard deviation. All experiments were repeated 3 times. ${ }^{* * *} \mathrm{P}<0.001$. NSCLC, non-small cell lung cancer; PTX, paclitaxel; BrdU, bromodeoxyuridine; DMSO, dimethylsulfoxide; IC50, half-maximal inhibitory concentration.

evaluated using a one-way analysis of variance. All statistical tests were performed with Prism software (version 5; GraphPad Software, Inc., La Jolla, CA, USA). P<0.05 was considered to indicate a statistically significant difference.

\section{Results}

MLN4924 exhibits a potent antiproliferative effect on PTX-resistant NSCLC cells. To investigate the effect of MLN4924 on A549/PTX and H460/PTXPTX-resistant NSCLC cells, the cells were treated with MLN4924 and used for several cell-based assays. As presented in Fig. 1A, MLN4924 induced the loss of NEDD8-conjugated (neddylated) cullins in A549/PTX and H460/PTX cells. In accordance with previous studies, cullin neddylation was substantially blocked in parent A549 and H460 cells following MLN4924 treatment (Fig. 1A) (21,22). BrdU assay analysis demonstrated that MLN4924 treatment led to a reduction in cell viability of A549/PTX and H460/PTX cells in a dose- and time-dependent manner (Fig. 1B). The half-maximal inhibitory concentration (IC50) values of A549/PTX were 

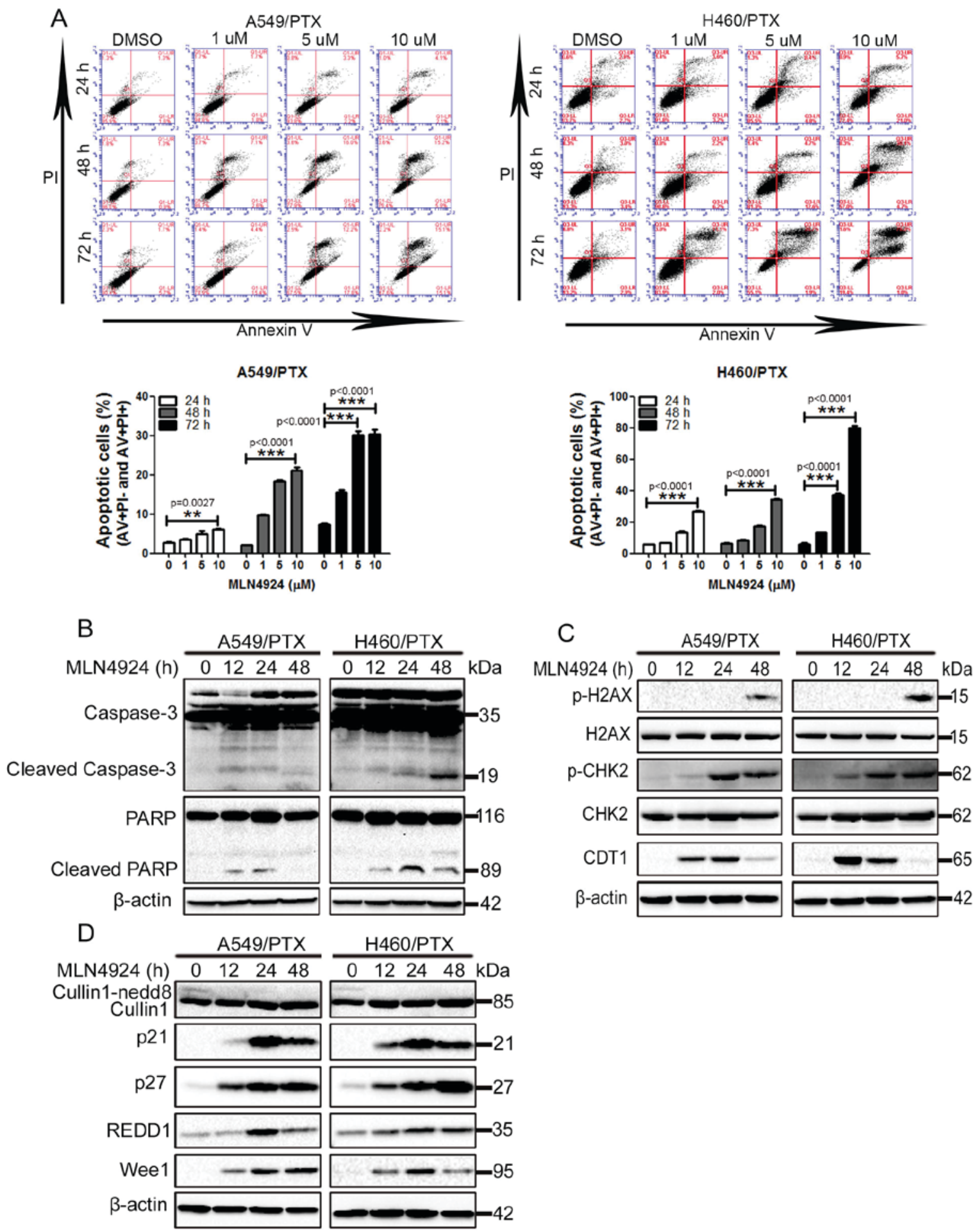

Figure 2. MLN4924 induces apoptosis and DNA damage in PTX-resistant non-small cell lung cancer cells. (A) A549/PTX and H460/PTX cells were treated with DMSO or 1, 5 and $10 \mu \mathrm{M}$ MLN4924 for 24, 48 and $72 \mathrm{~h}$. The cells were double-stained with AV and PI, apoptosis was analyzed using flow cytometry. The percentage of apoptotic cells were presented as the mean \pm standard deviation. The A549/PTX and H460/PTX cells were treated with vehicle or $10 \mu \mathrm{M}$ MLN4924 for 12, 24 and $48 \mathrm{~h}$. The cell lysates were collected and detected using western blot analysis. (B) Western blot analysis for caspase-3, PARP and $\beta$-actin. (C) Western blot analysis for p-H2AX, H2AX, p-CHK2, CHK2, CDT1 and $\beta$-actin. (D) Western blot analysis for cullin1, p21, p27, REDD1, Wee1 and $\beta$-actin. All experiments were performed 3 times. ${ }^{* *} \mathrm{P}<0.01,{ }^{* * *} \mathrm{P}<0.001$. PI, propidium iodide; AV, annexin V; PTX, paclitaxel; PARP, poly(ADP-ribose) polymerase; p-, phosphorylated; H2AX, histone 2AX; CHK2, checkpoint kinase 2; CDT1, chromatin licensing and DNA replication factor 1; REDD1, regulated in development and DNA damage responses 1.

$18.3,12.0$ and $7.3 \mu \mathrm{M}$ for 24,48 and $72 \mathrm{~h}$, respectively, and the IC50 values of H460/PTX were 12.9, 10.6 and $5.0 \mu \mathrm{M}$ for 24, 48 and $72 \mathrm{~h}$, respectively (Fig. 1B). Additionally, $10 \mu \mathrm{M}$ MLN4924 significantly decreased the clonogenic survival of
A549/PTX and H460/PTX cells ( $\mathrm{P}=0.0003$; Fig. 1C). Therefore $10 \mu \mathrm{M}$ MLN4924 was used throughout the current study. Taken together, these data indicate that MLN4924 potently inhibits the growth of A549/PTX and H460/PTX cells. 
A
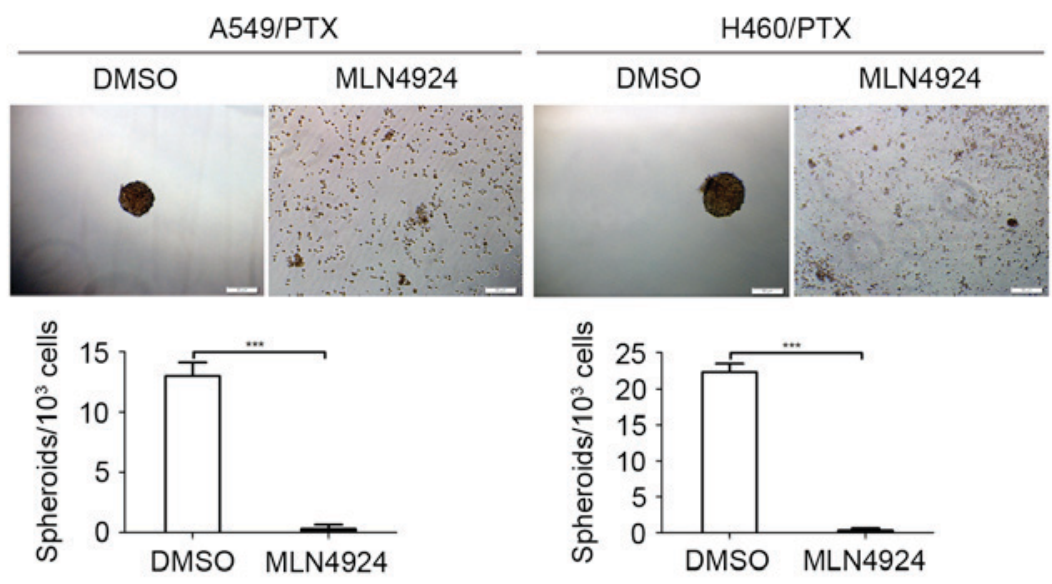

B
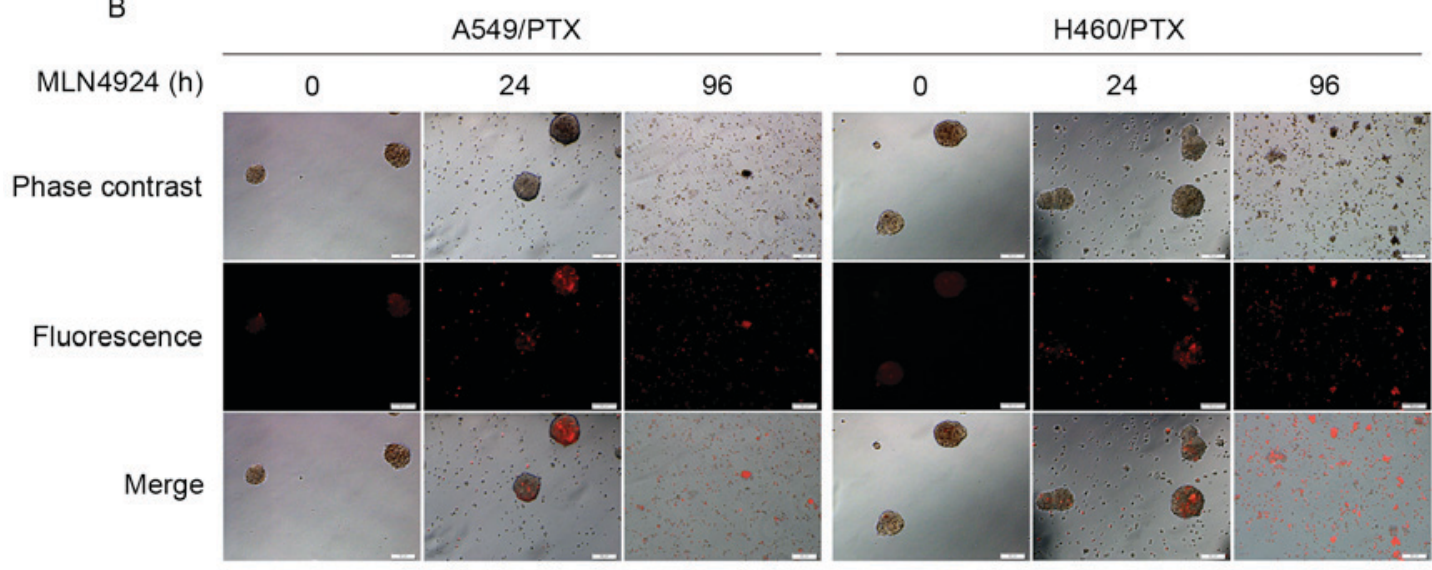

Figure 3. MLN4924 inhibits the growth of PTX-resistant non-small cell lung cancer cells in 3-dimensional cultures. (A) A549/PTX and H460/PTX cells were treated with DMSO or $10 \mu \mathrm{M}$ MLN4924 for $24 \mathrm{~h}$, and then cultured in ultra-low attachment plates for 10 days. The number of spheroids (diameter $\geq 50 \mu \mathrm{m})$ was counted and the results are expressed as the mean \pm standard deviation from three independent experiments. (B) A549/PTX and H460/PTX cells were cultured in ultra-low attachment plates for 10 days to form spheroids, the spheroids were treated with vehicle or $10 \mu \mathrm{M}$ MLN4924 for 24 and $96 \mathrm{~h}$ then stained with propidium iodide. The cells were captured using phase contrast and red fluorescence microscopy, and merged images were created. Scale bar, $50 \mu \mathrm{m}$. All experiments were performed 3 times. ${ }^{* * *} \mathrm{P}<0.001$. PTX, paclitaxel; DMSO, dimethylsulfoxide.

A

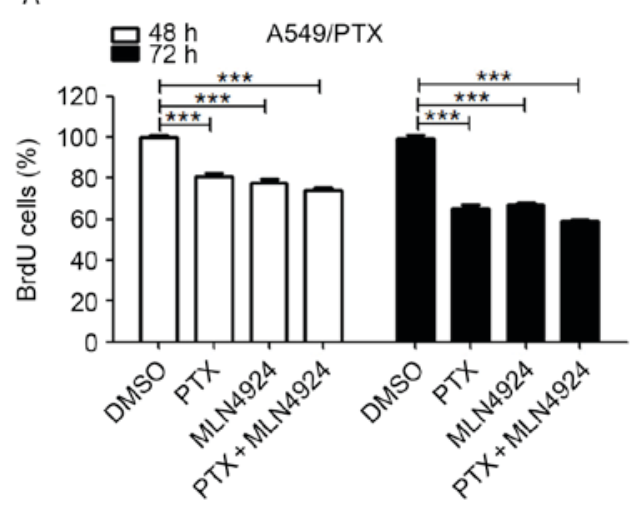

B

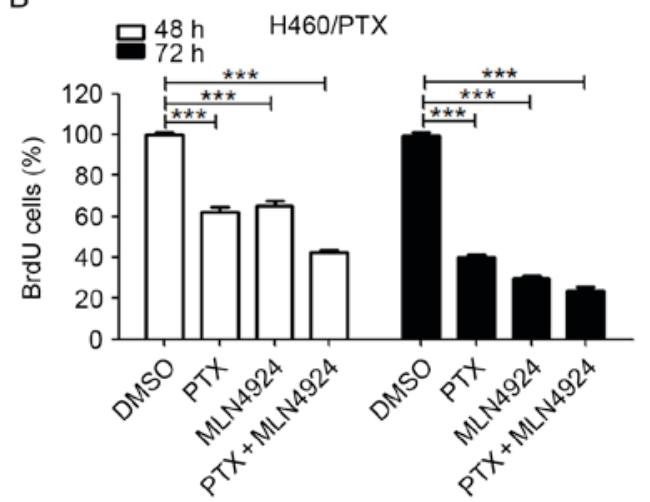

Figure 4. MLN4924 does not function synergistically with PTX in PTX-resistant non-small cell lung cancer cells. (A) A549/PTX and H460/PTX cells were treated with DMSO, $1 \mu \mathrm{M}$ PTX, $10 \mu \mathrm{M}$ MLN4924 or a combination of PTX and MLN4924 agents for 48 and $72 \mathrm{~h}$, and the cell viability was assessed using (B) BrdU assay. The results were quantified and presented as the mean \pm standard deviation. Experiments were performed 3 times. ${ }^{* * *} \mathrm{P}<0.001$. PTX, paclitaxel; DMSO, dimethylsulfoxide; BrdU, bromodeoxyuridine.

MLN4924 promotes apoptosis and DNA damage in PTX-resistant NSCLC cells. To investigate the underlying mechanism of how MLN4924 suppresses the growth of PTX-resistant NSCLC cells, MLN4924-treated A549/PTX and H460/PTX cells were analyzed for the induction of apoptosis using flow cytometry with annexin V and PI double staining, as MLN4924 has been established to induce apoptosis in a number of cancer cells $(11,15,22,23)$. As presented 
in Fig. 2A, exposure to MLN4924 led to a significant increase in apoptosis in A549/PTX and H460/PTX cells in a time- and dose-dependent manner. Furthermore, MLN4924 treatment promoted caspase-3 processing and PARP cleavage, 2 hallmarks of apoptosis, in A549/PTX and H460/PTX cells during a $48 \mathrm{~h}$ period (Fig. 2B). These results indicate that apoptosis is induced by MLN4924 in A549/PTX and H460/PTX cells.

In addition to inducing apoptosis, MLN4924 initiates the DNA damage response (DDR) in cancer cells $(8,11,13,23)$. Increased phosphorylation of histone $\mathrm{H} 2 \mathrm{AX}$ and $\mathrm{CHK} 2$, 2 classical markers of DDR, was observed in A549/PTX and H460/PTX cells following MLN4924 treatment (Fig. 2C). In addition, MLN4924-mediated accumulation of the DNA replication licensing factor, CDT1 in these cells (Fig. 2C). Taken together, these results indicate that MLN4924 promotes the DDR in PTX-resistant NSCLC cells.

It has been established that the effect of the induction of apoptosis and DDR by MLN4924 is due to its inactivation of CRL/SCF $(13,22-24)$. Therefore, the levels of numerous CRL/SCF substrates were examined in MLN4924-treated cells. As presented in Fig. 2D, MLN4924 treatment led to increased levels of p21, p27 and Wee1 in A549/PTX and H460/PTX cells, whereas cullin neddylation was inhibited. Increased levels of REDD1 were observed in MLN4924-treated A549/PTX and H460/PTX cells (Fig. 2D). These data indicate that MLN4924 efficiently inactivates CRL/SCF in A549/PTX and H460/PTX cells.

MLN4924 suppresses the growth of PTX-resistant NSCLC cells in 3-dimensional (3D) cultures. The 3D multicellular tumor spheroids are typically used as in vitro surrogates of tumorigenesis (25). Therefore, the growth inhibitory effect of MLN4924 was investigated in PTX-resistant NSCLC cells using 3D cultures. The A549/PTX and H460/PTX cells formed spheroids (with diameter of $\sim 50 \mu \mathrm{m}$ ) under appropriate condition for 3D cultures (Fig. 3A). However, MLN4924-treated A549/PTX and H460/PTX cells did not form spheroids compared with the DMSO-treated cells (Fig. 3A), indicating that MLN4924 has the ability to abrogate the 3D growth potential of the PTX-resistant NSCLC cells. Furthermore, following exposure to MLN4924 for $96 \mathrm{~h}$, the A549/PTX and H460/PTX spheroids had collapsed and there were increased levels of cell debris (Fig. 3B), indicating that prolonged treatment of MLN4924 promotes lysis of the A549/PTX and H460/PTX spheroids.

Combining MLN4924 with PTX does not exhibit synergy in PTX-resistant NSCLC cells. To investigate whether combining MLN4924 treatment with PTX results in an additive efficacy, the cell viability was evaluated using the BrdU assay. As presented in Fig. 4A, compared with each drug alone, the combination of MLN4924 and PTX treatment for numerous durations did not result in any significant alteration in cell viability in A549/PTX or H460/PTX cells. These data suggest that there is no synergistic effect between MLN4924 and PTX in the PTX-resistant NSCLC cells.

\section{Discussion}

Resistance to docetaxel or PTX remains a primary obstacle in the treatment of NSCLC. The current study demonstrated that the neddylation inhibitor, MLN4924, potently suppresses the growth of PTX-resistant NSCLC cells by inducing apoptosis and DNA damage. Furthermore, in addition to inducing clonogenic and spheroid formation, MLN4924 promotes the disassembly of PTX-resistant NSCLC cell spheroids. As MLN4924 is a first-in-class inhibitor of NAE that is being evaluated in multiple phase I clinical trials $(9,10)$. Soucy et al (14) identified that MLN4924 suppressed the growth of human tumor xenografts in mice, suggesting that NAE inhibitors may have potential as a treatment for cancer. The results of the present study provide a rationale for the clinical investigation of protein neddylation inhibition as a novel strategy for the treatment of PTX-resistant NSCLC.

An increasing number of studies have demonstrated that MLN4924 promotes a DNA damage response, cell cycle arrest, apoptosis and senescence in a number of cancer cell types $(8,11-13,15,22,23)$. In accordance with these previous studies, the current study observed that MLN4924 promotes apoptosis and the DDR in PTX-resistant NSCLC cells. Notably, MLN4924 suppresses the growth of PTX-resistant NSCLC cells in 3D culture. As the multicellular spheroids are an effective $3 \mathrm{D}$ cell culture model that is able to mimic in vivo microenvironments compared to 2D cell cultures (26), the results of the present study suggest a potential in vivo effect of MLN4924 on PTX-resistant NSCLC cells.

Consistent with previous studies (13,21-24), the effect of MLN4924 in PTX-resistant NSCLC cells was due to its inactivation of CRL/SCF demonstrated by the increased levels of a number of CRL substrates, including p21, p27, REED1 and Wee1. These data and the previous studies suggest an antineoplastic mechanism of action for MLN4924.

Previous studies have established that targeting NAE with MLN4924 effectively overcomes platinum resistance in preclinical models of ovarian cancer $(15,16)$. Additionally, a synergic cytotoxic effect between cisplatin and MLN4924 was observed in platinum-sensitive and -resistant ovarian cancer cells $(15,16)$. However, in the present study, combination treatment of MLN4924 and PTX did not result in synergistic cytotoxicity in the PTX-resistant NSCLC cells.

In conclusion, to the best of our knowledge, the current study is the first to demonstrate the growth inhibitory effect of MLN4924 in PTX-resistant NSCLC cells. The results of the present study support further investigation of NAE targeting with MLN4924 as an effective strategy for the treatment of PTX-resistant NSCLC.

\section{Acknowledgements}

The authors would like to thank Professor Songshu Meng (Institute of Cancer Stem Cell, Dalian Medical University of China, Liaoning, China) for critical discussions and suggestions. This study was supported in part by grants from the National Natural Science Foundation of China (grant nos. 81502674 to K.J. and 81301720 to W.C.).

\section{References}

1. Field JK and Duffy SW: Lung cancer screening: The way forward. Br J Cancer 99: 557-562, 2008.

2. Jemal A, Siegel R, Xu J and Ward E: Cancer statistics, 2010. CA Cancer J Clin 60: 277-300, 2010 
3. Ramalingam SS, Maitland ML, Frankel P, Argiris AE, Koczywas M, Gitlitz B, Thomas S, Espinoza-Delgado I, Vokes EE, Gandara DR and Belani CP: Carboplatin and Paclitaxel in combination with either vorinostat or placebo for first-line therapy of advanced non-small-cell lung cancer. J Clin Oncol 28: 56-62, 2010.

4. Joshi M, Liu X and Belani CP: Taxanes, past, present, and future impact on non-small cell lung cancer. Anticancer Drugs 25: 571-583, 2014

5. Xirodimas DP: Novel substrates and functions for the ubiquitin-like molecule NEDD8. Biochem Soc Trans 36: 802-806, 2008

6. Zheng N, Schulman BA, Song L, Miller JJ, Jeffrey PD, Wang P, Chu C, Koepp DM, Elledge SJ, Pagano M, et al: Structure of the Cul1-Rbx1-Skp1-F boxSkp2 SCF ubiquitin ligase complex. Nature 416: 703-709, 2002.

7. Jia L and Sun Y: SCF E3 ubiquitin ligases as anticancer targets. Curr Cancer Drug Targets 11: 347-356, 2011.

8. Brown JS and Jackson SP: Ubiquitylation, neddylation and the DNA damage response. Open Biol 5: 150018, 2015.

9. Sarantopoulos J, Shapiro GI, Cohen RB, Clark JW, Kauh JS, Weiss GJ, Cleary JM, Mahalingam D, Pickard MD, Faessel HM, et al: Phase I study of the investigational NEDD8-activating enzyme inhibitor pevonedistat (TAK-924/mlN4924) in patients with advanced solid tumors. Clin Cancer Res 22: 847-857, 2016.

10. Nawrocki ST, Griffin P, Kelly KR and Carew JS: MLN4924: A novel first-in-class inhibitor of NEDD8-activating enzyme for cancer therapy. Expert Opin Investig Drugs 21: 1563-1573, 2012.

11. Lin JJ, Milhollen MA, Smith PG, Narayanan U and Dutta A: NEDD8-targeting drug MLN4924 elicits DNA rereplication by stabilizing Cdt1 in S phase, triggering checkpoint activation, apoptosis, and senescence in cancer cells. Cancer Res 70: 10310-10320, 2010.

12. Mackintosh C, Garcia-Dominguez DJ, Ordóñez JL, Ginel-Picardo A, Smith PG, Sacristán MP and de Álava E: WEE1 accumulation and deregulation of S-phase proteins mediate MLN4924 potent inhibitory effect on Ewing sarcoma cells. Oncogene 32: 1441-1451, 2013.

13. Blank JL, Liu XJ, Cosmopoulos K, Bouck DC, Garcia K, Bernard H, Tayber O, Hather G, Liu R, Narayanan U, et al: Novel DNA damage checkpoints mediating cell death induced by the NEDD8-activating enzyme inhibitor MLN4924. Cancer Res 73: 225-234, 2013.

14. Soucy TA, Smith PG, Milhollen MA, Berger AJ, Gavin JM, Adhikari S, Brownell JE, Burke KE, Cardin DP, Critchley S, et al: An inhibitor of NEDD8-activating enzyme as a new approach to treat cancer. Nature 458: 732-736, 2009.

15. Jazaeri AA, Shibata E, Park J, Bryant JL, Conaway MR, Modesitt SC, Smith PG, Milhollen MA, Berger AJ and Dutta A: Overcoming platinum resistance in preclinical models of ovarian cancer using the neddylation inhibitor MLN4924. Mol Cancer Ther 12: 1958-1967, 2013
16. Nawrocki ST, Kelly KR, Smith PG, Espitia CM, Possemato A, Beausoleil SA, Milhollen M, Blakemore S, Thomas M, Berger A and Carew JS: Disrupting protein NEDDylation with MLN4924 is a novel strategy to target cisplatin resistance in ovarian cancer. Clin Cancer Res 19: 3577-3590, 2013.

17. Lv K, Liu L, Wang L, Yu J, Liu X, Cheng Y, Dong M, Teng R, Wu L, Fu P, et al: Lin28 mediates paclitaxel resistance by modulating $\mathrm{p} 21, \mathrm{Rb}$ and Let-7a miRNA in breast cancer cells. PLoS One 7: e40008, 2012.

18. Kim EH, Min HY, Chung HJ, Song J, Park HJ, Kim S and Lee SK: Anti-proliferative activity and suppression of P-glycoprotein by (-)-antofine, a natural phenanthroindolizidine alkaloid, in paclitaxel-resistant human lung cancer cells. Food Chem Toxicol 50: 1060-1065, 2012

19. Li Z, Jiang K, Zhu X, Lin G, Song F, Zhao Y, Piao Y, Liu J, Cheng W, Bi X, et al: Encorafenib (LGX818), a potent BRAF inhibitor, induces senescence accompanied by autophagy in BRAFV600E melanoma cells. Cancer Lett 370: 332-344, 2016.

20. Hu L, Sun S, Wang T, Li Y, Jiang K, Lin G, Ma Y, Barr MP, Song F, Zhang G and Meng S: Oncolytic newcastle disease virus triggers cell death of lung cancer spheroids and is enhanced by pharmacological inhibition of autophagy. Am J Cancer Res 5: 3612-3623, 2015

21. Li H, Tan M, Jia L, Wei D, Zhao Y, Chen G, Xu J, Zhao L, Thomas D, Beer DG and Sun Y: Inactivation of SAG/RBX2 E3 ubiquitin ligase suppresses KrasG12D-driven lung tumorigenesis. J Clin Invest 124: 835-846, 2014.

22. Li L, Wang M, Yu G, Chen P, Li H, Wei D, Zhu J, Xie L, Jia H, Shi J, et al: Overactivated neddylation pathway as a therapeutic target in lung cancer. J Natl Cancer Inst 106: dju083, 2014.

23. Luo Z, Yu G, Lee HW, Li L, Wang L, Yang D, Pan Y, Ding C, Qian J, Wu L, et al: The Nedd8-activating enzyme inhibitor MLN4924 induces autophagy and apoptosis to suppress liver cancer cell growth. Cancer Res 72: 3360-3371, 2012.

24. Gu Y, Kaufman JL, Bernal L, Torre C, Matulis SM, Harvey RD, Chen J, Sun SY, Boise LH and Lonial S: MLN4924, an NAE inhibitor, suppresses AKT and mTOR signaling via upregulation of REDD1 in human myeloma cells. Blood 123: 3269-3276, 2014

25. Loessner D, Flegg JA, Byrne HM, Clements JA and Hutmacher DW: Growth of confined cancer spheroids: A combined experimental and mathematical modelling approach. Integr Biol (Camb) 5: 597-605, 2013.

26. Pampaloni F, Reynaud EG and Stelzer EH: The third dimension bridges the gap between cell culture and live tissue. Nat Rev Mol Cell Biol 8: 839-845, 2007. 